\title{
Les enfants des milieux socioéconomiques défavorisés sont-ils massivement condamnés à l'échec scolaire?
}

Steve Bissonnette Université TELUQ (Canada)

\section{HRONIQUE • Formation des maitres}

Depuis plus d'une cinquantaine d'années, de nombreuses enquêtes sociologiques réalisées dans divers pays ont montré que lorsqu'on compare des enfants de milieux socioéconomiques défavorisés (SÉD) à ceux qui proviennent de milieux plus aisés, les enfants des milieux SÉD risquent davantage d'avoir des difficultés et des retards scolaires (Coleman et al., 1966; OECD, 2004). Des résultats comparables ont été observés au Québec.

Le ministère de l'Éducation, du Loisir et du Sport du Québec (MELS, 2005) a publié une étude qui analyse la réussite scolaire des élèves en fonction de leur milieu socioéconomique. Globalement, les résultats de cette enquête corroborent ceux présentés dans les diverses études sociologiques citées précédemment. Les constats de ces différentes enquêtes sociologiques, largement diffusés, contribuent malheureusement à alimenter la croyance que l'école et l'enseignant ont peu d'impact sur la réussite scolaire des élèves provenant de milieux SÉD. Est-ce vraiment le cas?

\section{L'effet école}

Malgré le lien existant entre les difficultés scolaires de l'élève et son statut socioéconomique, on ne peut conclure que l'ensemble des élèves issus d'un milieu SÉD auront des difficultés ou des retards scolaires (MELS, 2005). Collerrette et Pelletier (2017) observent auprès de 11 commissions scolaires québécoises (15 000 élèves), une " relation statistique effective entre l'indice de défavorisation et la proportion d'élèves vulnérables ${ }^{1}$ dans l'ensemble des commissions scolaires " (p.14). Cependant, les deux chercheurs indiquent que «l'indice de défavorisation sociale n'explique pas tout et d'autres facteurs de risque et de protection sont à l'œuvre » (p. 15). En effet, leurs résultats 
démontrent qu'il y a plusieurs écoles ayant un indice de défavorisation relativement élevé dont les élèves réussissent nettement mieux que d'autres écoles ayant un indice de défavorisation comparable. De plus, certaines écoles « défavorisées » obtiennent de meilleurs rendements que des écoles ayant un indice socioéconomique plus favorable. Par conséquent, les chercheurs concluent que « les données recueillies ici tendent à démontrer la présence d'un effet école dans l'ensemble des commissions scolaires de l'échantillon » (p. 15). Si certaines écoles ont un effet positif indéniable sur le rendement des enfants des milieux SÉD, il est important de souligner que l'effet de l'enseignant sur le rendement des élèves est encore plus puissant.

\section{L'effet enseignant}

Des méga-analyses, comme celles produites par Hattie (2012), indiquent clairement l'effet massif de l'enseignement et de l'enseignant. D'autres études mesurant la valeur ajoutée de l'enseignant réalisées par Sanders et Rivers (1996) comparent de manière plus fine l'impact de différents facteurs sur le rendement des élèves. L'ensemble de ces recherches ont montré l'effet déterminant de l'enseignant auprès des élèves qui éprouvent des difficultés scolaires. Cet effet enseignant est même supérieur à ceux de l'école et de la famille sur le rendement des élèves. Malgré la forte convergence des recherches montrant l'effet enseignant, celles-ci ont fait l'objet de sérieuses critiques sur le plan méthodologique (Bergeron, 2016; Wiliam, 2018). Pour parer aux limites de ces études, Wiliam (2018) analyse l'effet enseignant à la lumière des recherches menées sur les " caractéristiques qualitatives des enseignants » (teacher quality). Dans ce type de recherche, l'effet enseignant est révélé en comparant les progrès des élèves entre le début et la fin d'une année scolaire, à l'aide d'une épreuve standardisée (Wiliam, 2018).

Hanushek (1971) a été l'un des premiers chercheurs à étudier les caractéristiques qualitatives des enseignants. Hanushek a observé que les progrès des élèves sur une année scolaire variaient considérablement entre les classes. Il a montré que les élèves dans certaines classes pouvaient faire jusqu'à cinq mois de progrès de plus que des élèves comparables d'autres classes, ce qui représente une différence plus que considérable.

Aaronson, Barrow et Sander (2007) analysent les caractéristiques qualitatives d'enseignants de $9^{\mathrm{e}}$ année dans les écoles publiques de Chicago sur la base des progrès de leurs élèves au cours d'une année scolaire. Sur cette base, les enseignants ont été divisés en trois catégories : très efficaces, moyennement efficaces et peu efficaces. Or, les élèves assignés aux enseignants très efficaces font $40 \%$ plus de progrès sur une année scolaire que ceux confiés aux enseignants moyennement efficaces tandis que les élèves assignés aux enseignants peu efficaces font $30 \%$ moins de progrès que ceux confiés aux enseignants moyennement efficaces. En d'autres mots, le progrès des élèves assignés aux enseignants très efficaces est deux fois plus élevé que celui des élèves confiés aux enseignants peu efficaces.

Rockoff (2004) examine les caractéristiques qualitatives d'enseignants du primaire sur une période de 12 années dans deux districts du New Jersey. Les progrès annuels des élèves étant mesurés en lecture et en mathématiques, on constate que, même sur une longue période, certains enseignants sont toujours plus efficaces que d'autres. Dans cette étude, les élèves confiés aux enseignants très efficaces font $50 \%$ plus de progrès en mathématiques et $40 \%$ plus de progrès en lecture que ceux confiés aux enseignants moyennement efficaces. 
Finalement, Kane, McCaffrey, Miller et Staiger (2013) ont montré que des enseignants identifiés comme très efficaces dans une école, puis mutés l'année suivante dans une autre école et devant enseigner à un autre niveau, et ce, auprès d'autres élèves provenant de quartiers différents, sont toujours identifiés comme très efficaces la seconde année.

Par conséquent, les études sur l'effet école et celles sur l'effet enseignant convergent toutes dans le même sens, malgré leurs différences et leurs limites méthodologiques : l'école et les enseignants ont une influence déterminante sur la réussite des élèves qui leur sont confiés et, en corollaire, non, il n’y a pas que le milieu socioéconomique qui joue dans la réussite scolaire des enfants.

\section{Note}

1 Une catégorie d'élèves vulnérables est constituée d'élèves ayant obtenu des résultats de $69 \%$ et moins à l'ensemble des quatre épreuves ministérielles combinées de la $6^{\mathrm{e}}$ année $:$ une épreuve en lecture, une en écriture et deux en mathématiques.

\section{Références}

Aaronson, D., Barrow, L. et Sander, W. (2007). Teachers and student achievement in the Chicago public high schools. Journal of Labor Economics, 25(1), 95-135. http://dx.doi.org/10.1086/508733

Bergeron, P.-J. (2016). Comment faire de la pseudoscience avec des données réelles : une critique des arguments statistiques de John Hattie dans Visible Learning par un statisticien. McGill Journal of Education, 51(2), 935-945. http://dx.doi.org/10.7202/1038611ar

Coleman, J. S., Campbell, E. Q. Hobson, C. J., McPartland, J., Mood, A. M., Weinfield, F. D. et York, R. L. (1966). Equality of educational opportunity. Repéré à https://files.eric.ed.gov/fulltext/ED012275.pdf

Collerrette, P. et Pelletier, G. (2017). Les résultats des élèves finissants du primaire de 13 commissions scolaires aux épreuves du MELS en juin 2016 et 2017 (Rapport de recherche). Université du Québec en Outaouais.

Hanushek, E. A. (1971). Teacher characteristics and gains in student achievement: Estimation using micro data. American Economic Review, 61(2), 280-288. Repéré à http://hanushek.stanford.edu/publications/teacher-characteristics-and-gainsstudent-achievement-estimation-using-micro-data

Hattie, J. A. (2012). Visible learning for teachers. Maximizing impact on learning. New York, NY : Routledge.

Kane, T. J., McCaffrey, D. F., Miller, T. et Staiger, D. O. (2013). Have we identified effective teachers? Validating measures of effective teaching using random assignment. Repéré à https://files.eric.ed.gov/fulltext/ED540959.pdf

Ministère de l'Éducation, du Loisir et du Sport (MELS). (2005). La réussite scolaire des garçons et des filles. L’influence du milieu socioéconomique. Analyse exploratoire. Repéré à http://www.education.gouv.qc.ca/fileadmin/site web/documents/dpse/ Brochure reus influence ISBN2550441443.pdf

OECD. (2004). Learning for tomorrow's world: First results from PISA 2003. Paris : OECD. http://dx.doi.org/10.1787/9789264006416-en

Rockoff, J. E. (2004). The impact of individual teachers on student achievement: Evidence from panel data. American Economic Review, 94(2), 247-252. http://dx.doi.org/10.1257/0002828041302244

Sanders, W. L. et Rivers, J. C. (1996). Cumulative and residual effects of teachers on future student academic achievement. Repéré à https://www.heartland.org/ template-assets/documents/publications/3048.pdf

Wiliam, D. (2018). Creating the schools our children need. West Palm Beach, FL : Learning Sciences International.

\section{Pour citer cet article}

Boyer, C. et Bissonnette, S. (2019). Les enfants des milieux socioéconomiques défavorisés sont-ils massivement condamnés à l'échec scolaire?. Formation et profession. 27(2), 115-117. http://dx.doi.org/10.18162/fp.2019.a174 